\title{
The Impact of Motivator and Demotivator Factors on Agile Software Development
}

\author{
The Case of Pakistan
}

\author{
Shahbaz Ahmed Khan Ghayyur, \\ Salman Ahmed \\ Department of Computer Sciences \\ and Software Engineering, \\ International Islamic University \\ Islamabad, Pakistan
}

\author{
Saeed Ullah \\ Department of Computer Science \\ Federal Urdu University of Arts, \\ Science and Technology \\ Islamabad, Pakistan
}

\author{
Waqar Ahmed \\ Department of Statistics, \\ Comsats University Lahore, \\ Lahore, Pakistan
}

\begin{abstract}
Since the last decade, Agile software development has emerged as a widely utilized software development method keeping in view the developing countries of South Asia. The literature reports significant challenges and barriers for agile in software industry and thus the area still has significant problems when considered with this domain. This study reports an industrial survey in Pakistani software industry practices and practitioners to elicit the indigenous motivator and demotivators of agile paradigm in Pakistan. This study provides a concrete ranking of motivator and demotivator factors which influence the agile paradigm. A lack of proper training and other identified issues indicate that the adoption of agile is in preliminary phases and serious effort is required to set the direction right for success of agile paradigm and its adopting institutions. The survey is conducted in 23 companies practicing agile organizations and involves 90 agile practitioners. Reports of 67 practitioners were finally selected after careful selection against selection criteria for this study. The results indicate various alarming factors which are different from reported literature on the subject. Tolerance to work is the most important motivating factor among Pakistan agile practitioners, likewise lack of resources is the highest demotivating factor. A detailed ranking list of motivators and demotivators and comprehensive data analysis has been provided in this paper which influences strongly the agile software development issues in Pakistan.
\end{abstract}

Keywords-Agile software development; motivators; demotivators; success factors; barriers; agile methods; software development life cycle

\section{INTRODUCTION}

Agile software development is a repetitive method to produce acute and disciplined software development. Research study suggest that agile is the mostly used software development technique all over the world but in under developing countries due to their less domain knowledge and lack of experience there exist many barrier to proper implementation of agile methods [1]. As there is new shift of software industry from SDLC to Agile there exist many individuals and collective problem which caused hurdle in implementation of agile methodology [2]. These hurdles exist in individual and communal manner. To gauge these issues, a survey has been conducted in Pakistan to check the impact of motivators and demotivator factors in agile software development in Pakistani Software industry. This survey will help to enhance productivity of software and reduced number of demotivator factors [3].

In Pakistan, Agile is nourished as Emerging field. In past decade, due to the lack of interest and strategy of software practitioners, software industry face many economical issues but as the agenda of agile become popular there following increase immensely [4]. The formation of PSEB is also an initiative to ensure assistance of software industry. In recent years, Agile boom has become a latest trend in software industry. According to PSEB, about $70 \%$ of software organization has converted or thinking to convert their development method on Agile.

In ASD, due to their iterative nature has less failure ratio than SDLC. For this reason, many organizations local or international software industry moving towards agile due to their well-defined set of rules and well organization teams [5]. Motivators factors in agile plays a key role in development of agile industry. These motivating factors provide ability to work on self-determination and to made better product.

As the most common concern for an organization is to provide productive software to their end-users and this phenomena is only achieve by providing motivation to their employee and to avoid the demotivator factor as possible [6]. Motivators plays important role in enhancing people and technical skills. A lot of work has been done to motivator SDLC practitioners but agile has less contribution in this regard [7].

\section{A. The Need of Empirical Analysis}

As agile is mostly used method in software industry, but it requires a lot of work on employee's motivation for their full adaption. The main concern is to remove the practitioners' anxiety for adoption of agile method. In this regard, motivator and demotivator factors play a critical role [8]. These factors can be used to alter the software productivity and these factors can provide new power to agile industry.

In Pakistan, software industry is growing day by day but due to the higher failure ratio of projects is become a worry sign for software development organization. For this reason, 
they want to trade towards agile but due to the barriers exist in the form of demotivator factors they can't fully yield the concern results. Due to which a survey study is conducted to gauge the concern of agile practitioners. For this purpose, motivator and demotivator factors has been collected from literature and a survey is been conducted which rank these motivators and demotivators according to Pakistan software industry.

This study will also contribute to gather the motivator factors of agile, which is present in dispersed form and need to analyse. The literature is mainly covering the motivator factor of SDLC but agile is neglected. There exist a gap to empirically analysis of the motivator and demotivator factors [9]. By finding these motivator factors, list of demotivator factors can reduced which eventually result in quality software product.

The arrangement of this article is ordered as: Section II briefly describe the literature review regarding motivators and demotivators factors. Section III explains the research methodology used during research. Section IV describes the indetail analysis and results regarding survey. Research contribution is discussed in Section V, Section VI is covering portion of results and discussion and finally Section VII describe conclusion and future work.

\section{RELATED WORK}

Motivators and Demotivators has a vital role in software productivity. This portion will provide a brief literature review of work done in motivators and demotivators of Agile Software Development. De O et al. [10] provides a detailed list of motivators and demotivators in software development life cycle. Afterward they propose model of motivation of software Engineering(MOCC) in which they divide the motivator into different category .Highsmith and Cockburn [11] are the member of agile formation team, they provide the benefit of adopting agile software development. Akhtar et al. [12] has conducted a survey in Pakistani software industry about the barrier exist in Scrum method, there findings suggest that there exist many flaws in full fledge implementation of Scrum in Pakistani software industry. Hassan et al. [13] briefly describe the challenges exist in full adoption of scrum in Pakistan. There finding suggest that scrum is newly implemented in Pakistan that's why they require adequate training for their full implementation. Wagener [14] listed down detailed list of motivators and demotivators in SDLC afterward they categorize the motivator factor into three groups: organizational, people and technical. Chow and Cao [15] has conducted survey among 109 Agile Teams among different organization, on the basis of survey they find new motivator factor of agile software development. Baddoo and Hall [16] has done a detailed analysis among SDLC factors in which they have found that rewards and incentive to employees can increase their productivity. Asghar and Usman [17] has done a Systematic literature review of Motivators and Demotivators of Software development life cycle, they proposed a model of motivation for Pakistan industry in which they claim Hofstede's cultural issue is the biggest barrier in this region.

\section{RESEARCH METHOD DETAIL}

Due to the limitation in research and difference in survey method, mail method in questionnaire and personal method are selected. Research questions are shown in Table I.

\section{A. Research Questions}

TABLE I. RESEARCH QUESTIONS

\begin{tabular}{|l|l|l|}
\hline Sr.no & Research Questions & Motivation \\
\hline 1 & $\begin{array}{l}\text { What are the motivator } \\
\text { and de-motivator factors } \\
\text { existed in Agile Software } \\
\text { Development? }\end{array}$ & $\begin{array}{l}\text { This question will provide a detailed } \\
\text { list of motivators and demotivators } \\
\text { of agile. }\end{array}$ \\
\hline 2 & $\begin{array}{l}\text { What are the impact of } \\
\text { motivators and } \\
\text { demotivators on software } \\
\text { industry? }\end{array}$ & $\begin{array}{l}\text { This question aims to provide a } \\
\text { detailed discussion of impact of } \\
\text { motivators and demotivators in } \\
\text { software industry. }\end{array}$ \\
\hline
\end{tabular}

B. Questionaire Design

The Questionnaire is divided into three sections

Section 1: Include Respondents profile.

Section 2: Include company's profile.

Section 3: Include Motivator and Demotivator of Agile.

\section{Data Collection Techniques and Methodologies}

Questionnaire is floated via two methods, email and Personal contacts. In both these medium our target is those organization who are fully or partially practices agile. For this purpose, list of organizations has taken from PASHA and PSEB and try to target maximum population. Along with pass on strategy personal contacts also been made to target more organization following agile. A total of 25 software organizations were visited. A total of 25 companies were chosen to provide the research method with pre-requisite to following agile fully or partially.

\section{Sample}

The whole population (23 agile companies) employees were the sample of the study. As we have limited sample size that's why regular follow ups with respondents containing email and telephone calls and meetings are arranging to get the maximum number of respondents. Some appreciation cards and other incentive are also arranging to get the maximum number of accurate Reponses.

\section{E. Identification of Agile Practitioners}

In this survey, one thing is assured that all respondents must possess agile background. The background considers regarding agile will be fully and partially usage. For this purpose, companies are visited personally plus email and phone are used to convince agile practitioners to fill the survey. Cross questioning has been made to verify the respondent's record to double check the practitioner knowledge regarding agile. 
Identification of agile practitioner is check using three steps:

1) Respondents are currently working / have already worked at organization which practices agile.

2) Respondents are currently working / have already worked in an organization where at least one agile method is used, e.g. Scrum, Kanban, etc.

3) Respondent must be willing to give interview in given time slot.

\section{F. Compilation of Issues}

Once the feedback of survey is received, compilation work has been started. For this purpose, two software Microsoft Excel and SPSS (Version 24) are used to get better view of respondent's behaviour towards the survey. After accessing all feedback responses, a list of issue was extracted based on respondents output.

\section{G. Interview to Resolve Open Issues}

Interview is conducted to address some open issues which can't be address in questionnaire. A session of two interviews with practitioners using agile is conducted in which open issue are briefly discussed. The opinion is included in conclusion.

\section{H. Identification of Renowned Agile Practitioners}

Selection of renowned agile practitioners has been collected on following criteria:

1) At least five years of agile experience.

2) Worked in an organization using agile more than two years.

3) Taken and conducted agile trainings in past two years.

4) Achieve agile certifications.

\section{Compilation of Data (Interview and Survey Result)}

A total number of 25 companies were visited. Participating companies were selected from given number of respondents give the information about the motivator and demotivator of Agile and different initiative to reduce the demotivator factors. The companies were chosen to provide the cross section of current profile, total working experience, experience usage of agile method, extend of usage of agile method and preference of most using agile practices.

\section{J. Analysis Method Used}

There are two major analysis one is qualitative and other one is quantitative. Both techniques are used to measure the more accuracy of respondent's feedback.

\section{K. Quantitative Analysis}

Quantitative analysis is best used analysis technique to measure the respondents result more accurately. In quotative analysis rather than question and their answer numeric data is prominent by which significant of research is prominent. Our focus is to target the quantitative analysis to get a more accurate result with respect to motivator and demotivator of agile. Table II shows the key aggregate on Surveys response.
TABLE II. KEY AGGREGATE ON RESPONSE

\begin{tabular}{|c|c|}
\hline Total Number of Software Companies Surveyed & 25 \\
\hline Total Number of Software companies using agile & 23 \\
\hline Companies working on Offshore Development: & 14 \\
\hline Companies working on In-house Development: & 07 \\
\hline Companies working on Both: & 02 \\
\hline Small-Medium Companies: & 14 \\
\hline Large Companies: & 09 \\
\hline Total Number of Software Practitioners Contacted & 90 \\
\hline Total Number of Software Practitioners Responde & 67 \\
\hline Respondent's Total Experience (3-5 Yrs): & 20 \\
\hline Respondent's Total Experience (1 to >3 Yrs): & 22 \\
\hline Respondent's Total Experience (5-10 Yrs): & 10 \\
\hline Respondent's Total Experience (10> Yrs): & 15 \\
\hline Business Analyst/ Professional services & 02 \\
\hline Project Management & 09 \\
\hline Team Lead & 4 \\
\hline Junior Software Developer & 07 \\
\hline Senior Software Developer & 32 \\
\hline Software test Engineer & 05 \\
\hline Quality Assurance & 08 \\
\hline Total Number of Questions in Questionnaire & 19 \\
\hline Mandatory Questions: & 16 \\
\hline Optional Questions: & 03 \\
\hline Total Number of Strongly Agreed Responses & 427 \\
\hline Number of Agreed Responses: & 1525 \\
\hline Number of Neutral Responses: & 425 \\
\hline Number of Disagreed Responses: & 167 \\
\hline Number of Strongly Disagree & 23 \\
\hline
\end{tabular}

\section{ANALYSIS AND RESUlts}

Author has already study the motivator and demotivator factors and identified issue according to agile software development and categorize into three factors: People, technical and organization. The same motivator and demotivator factors are used in a survey conducted in Pakistani Agile Software industry. The aim of this survey is to find out the higher rank motivator and demotivator factors and then results shown below is used to find out the issues of agile practitioners and compare results with the literature to increase the motivator factors in ASD. 
TABLE III. CRONBACH'S ALPHA FOR PILOT STUDY

\begin{tabular}{|l|l|l|}
\hline Scales & K & Cronbach's Alpha ( $\boldsymbol{\alpha})$ \\
\hline Motivation factors & 36 items & 0.895 \\
\hline Demotivation factors & 24 items & 0.923 \\
\hline
\end{tabular}

To check the reliability of survey, Cronbach alpha test is applied. Motivator contain 36 factors whereas demotivator contains 24 factors, Cronbach alpha test shows that both values are highly reliable.

\section{Cronbach on survey}

Table III shows reliability analyses of scales used by the motivation factor and demotivation factors, $\alpha=.895$ and $\alpha=$ .923 respectively.

\section{A. Profile of Respondents}

1) Gender based respondents: Empirical analysis result shows that male respondents are more than female respondents. They have 59 and 8 frequencies respectively.

Following Fig. 1 shows the gender respondents of pilot study.

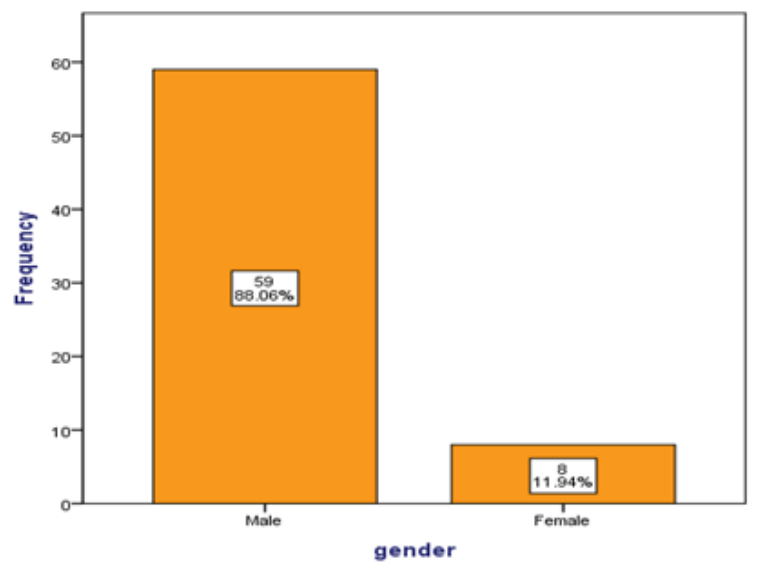

Fig. 1. Pilot study gender wise respondents.

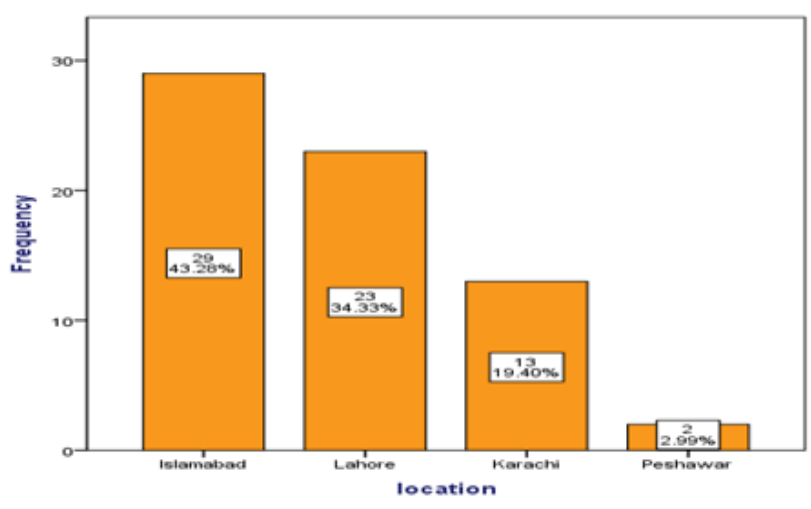

Fig. 2. Cities wise respondents.
2) Location based respondents: Islamabad has more frequency of respondents than other cities of Pakistan. Its frequency is 29 whereas Lahore has 23, Karachi has 13 and Peshawar has 2 respondent's frequencies.

Fig. 2 shows the cities by which responders fill the pilot study.

\section{B. Current Profile based Respondents}

According to our respondent's Senior software developers has more number of respondent's frequency which is 32 whereas project Managers has 9, Quality assurance engineer has 8, Junior software developer has 7, software test engineer has 5, team lead has 4 and Business analyst has 2 respondents.

The following Fig. 3 shows the total experience of responders

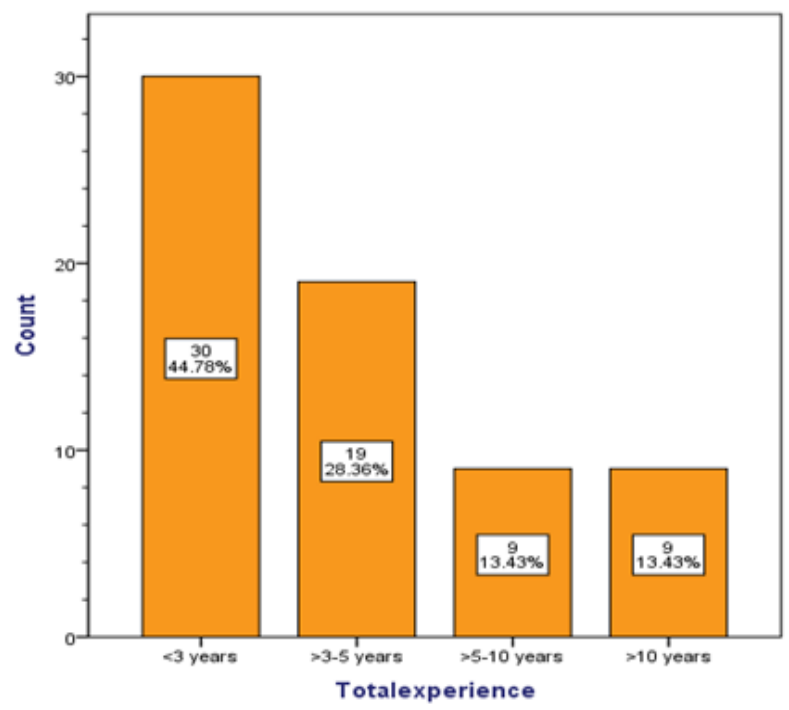

Fig. 3. Respondents total experience.

\section{Agile Experience of Respondent's}

The result indicates that the respondents having 1 to less than three years' experience are $43 \%$, while less than one year has $30 \%$, the experience from 3 to 5 years are $14 \%$, the respondent's having experience from less than 5 to 10 years are $9 \%$ and the respondents having experience more than 10 years are $4 \%$.

Following Fig. 4 is depicted the agile experience of respondents.

Following Table IV shows the demographic profile of respondents based on gender, location, current profile, total experience and agile experience. To make better understanding of results, the results are shown in frequency as well as percentage.

\section{Extend of Usage of Agile Methods}

The following Fig. 4 shows the responders usage of agile method to different type of project. The results indicate that agile is been used for majority of projects and is using large number in organizations ongoing projects. 


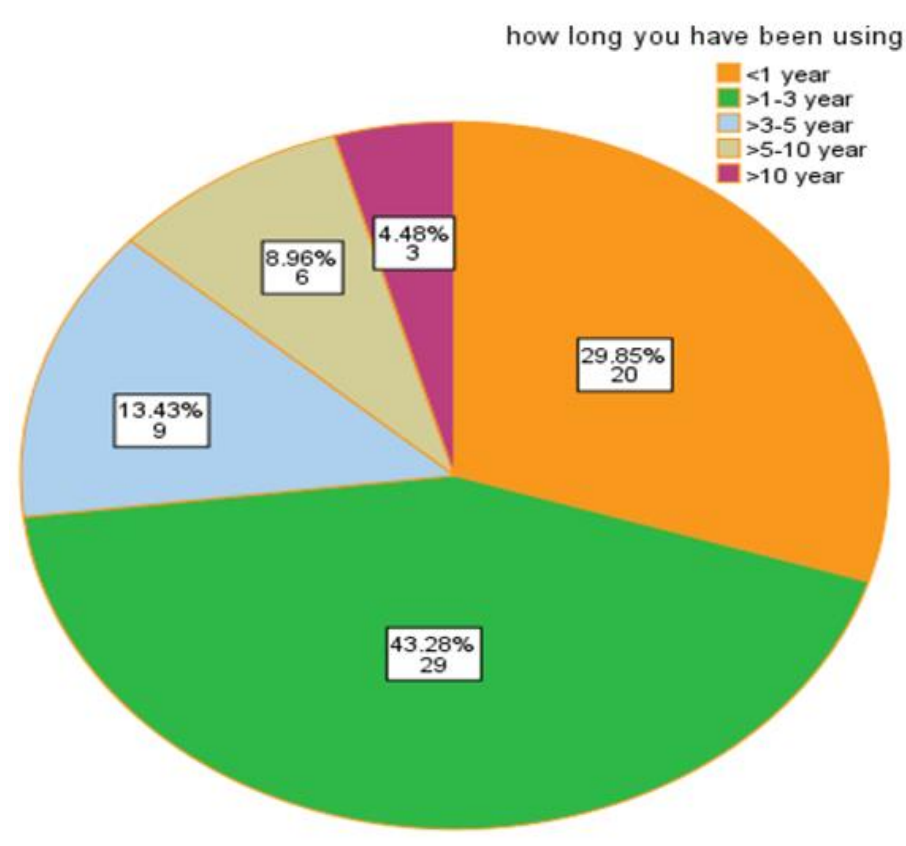

Fig. 4. Duration of agile usage.

TABLE IV. Socio-Demographic Profile of PILOT StUdy

\begin{tabular}{|c|c|c|}
\hline \multicolumn{2}{|l|}{ Category } & Frequency $(\%)$ \\
\hline$C$ & Male & $59(88.1)$ \\
\hline Uеताuटt & Female & $8(11.9)$ \\
\hline \multirow{4}{*}{ Location } & Islamabad & $29(43.3)$ \\
\hline & Lahore & $23(34.3)$ \\
\hline & Karachi & 13(19.4) \\
\hline & Peshawar & $2(3.0)$ \\
\hline \multirow{7}{*}{ Describe your current profile } & Business Analyst/ Professional services & $2(3.0)$ \\
\hline & Project Management & $9(8.9)$ \\
\hline & Team Lead & $4(6.0)$ \\
\hline & Junior Software Developer & $7(10.4)$ \\
\hline & Senior Software Developer & $32(47.8)$ \\
\hline & Software test Engineer & $5(7.5)$ \\
\hline & Quality Assurance & $8(11.9)$ \\
\hline \multirow{4}{*}{ Total Work experience } & $<3$ years & $30(44.8)$ \\
\hline & 3 years to 5 years & $19(28.4)$ \\
\hline & $>5$ years to 10 years & $9(13.4)$ \\
\hline & $>10$ years & $9(13.4)$ \\
\hline \multirow{5}{*}{ How long you have been using Agile methods } & Less than a Year & 20(29.9) \\
\hline & $1-3$ years & $49(43.3)$ \\
\hline & $>3$ years to 5 years & $9(13.4)$ \\
\hline & $>5$ years to 10 years & $6(9.0)$ \\
\hline & $>10$ years & $3(4.5)$ \\
\hline
\end{tabular}




\section{E. Usage of Agile Methods}

Fig. 5 shows the respondents answer of using different agile methods. The result indicates that Scrum is mostly used method with usage of $20 \%$, extreme programming has usage of $11 \%$ and crystal-clear method has least usage of $1 \%$.

On a question of most using agile practice, Fig. 6 the respondents agree on planning iteration with $55 \%$, daily stand ups have $22 \%$, Iteration retrospective has $4.5 \%$ and Review meeting has $3 \%$ usage in respondent's organizations.

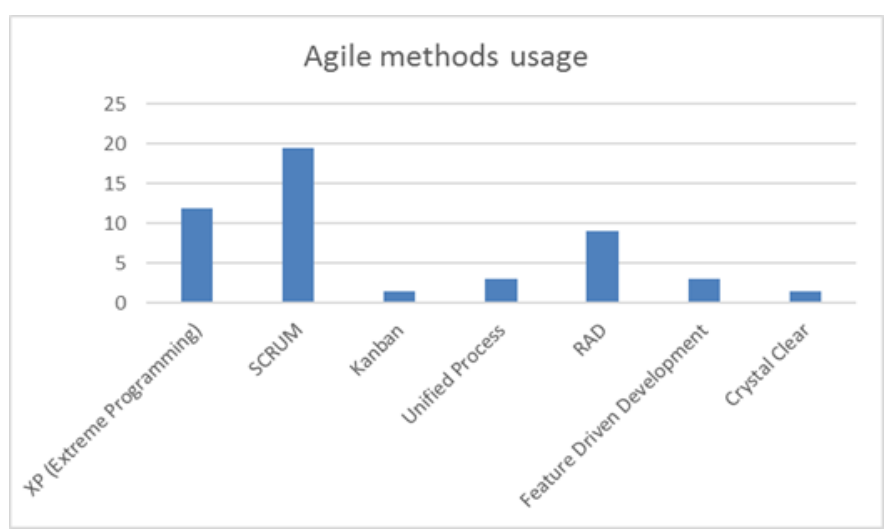

Fig. 5. Agile usage method usage of agile practices.

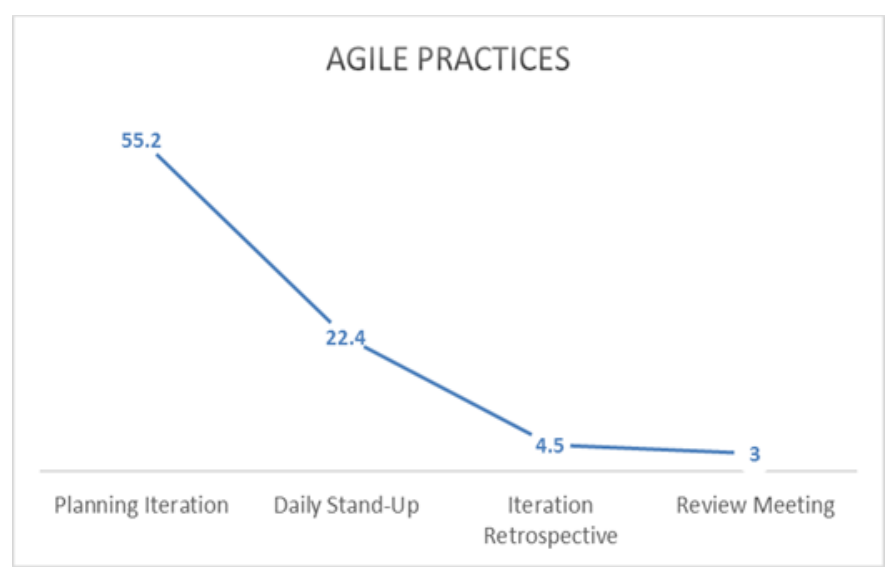

Fig. 6. Agile practices usage.

Following Table $\mathrm{V}$ shows the information about the agile usage in which first block is answering about extend of usage of agile methods in majority, large, small and other projects. In most fluent agile method scrum has 19\%, extreme programming has $20 \%$, and crystal clear has least $1 \%$ agile method. In preference of agile method, the core agile practices such as planning iteration, Daily Stand-ups, Iteration retrospective and review meeting.

TABLE V. AgILE Method you are Fluent Most (Pilot Study)

\begin{tabular}{|c|c|c|}
\hline \multicolumn{2}{|l|}{ Category } & \multirow{2}{*}{$\begin{array}{l}\text { Frequency }(\%) \\
32(47.8) \\
\end{array}$} \\
\hline \multirow{6}{*}{ Extend of Agile Methods } & Majority of Projects & \\
\hline & Large number & 13(19.4) \\
\hline & Small number & $10(14.9)$ \\
\hline & Just Started & $2(3.0)$ \\
\hline & In learning phase & $9(13.4)$ \\
\hline & Have never used & $1(1.5)$ \\
\hline \multirow{10}{*}{ In which Agile method you are fluent most } & XP (Extreme Programming) & $8(11.9)$ \\
\hline & SCRUM & $13(19.4)$ \\
\hline & Kanban & $1(1.5)$ \\
\hline & Unified Process & $2(3.0)$ \\
\hline & RAD & $6(9.0)$ \\
\hline & Feature Driven Development & $2(3.0)$ \\
\hline & Crystal Clear & $1(1.5)$ \\
\hline & Team Software Process & $2(3.0)$ \\
\hline & Agile Modeling & $7(10.4)$ \\
\hline & N/A & $3(4.5)$ \\
\hline \multirow{4}{*}{ Preference of Agile Practice } & Planning Iteration & $37(55.2)$ \\
\hline & Daily Stand-Up & $15(22.4)$ \\
\hline & Iteration Retrospective & $3(4.5)$ \\
\hline & Review Meeting & $2(3.0)$ \\
\hline
\end{tabular}




\section{F. Respondents Responses}

1) Motivators responds: Following Table VI are the motivators ranking evaluated by the respondent's results.
According to which tolerance to work has most $98 \%$ while the eliminated managerial politics is the least number of motivator with $25 \%$.

\section{TABLE VI. RESPONDENTS MOTIVATORS RESUlT}

\begin{tabular}{|c|c|c|c|c|}
\hline Motivating Factors & Strongly Agree & Agree & Disagree & Strongly Disagree \\
\hline Rewards and incentives & 21 & 35 & 0 & 2 \\
\hline Management Supportive role & 21 & 35 & 4 & 0 \\
\hline Well defined coding standard & 15 & 30 & 2 & 0 \\
\hline Career path & 11 & 43 & 0 & 0 \\
\hline Better working environment & 20 & 42 & 2 & 0 \\
\hline Variety of work & 14 & 35 & 5 & 0 \\
\hline Technically challenging work & 10 & 36 & 1 & 2 \\
\hline Successful company experience & 11 & 36 & 2 & 1 \\
\hline Trust & 20 & 30 & 3 & 0 \\
\hline Identify with the task & 11 & 36 & 1 & 1 \\
\hline Sufficient resources & 8 & 32 & 2 & 0 \\
\hline Development needs addressed & 13 & 40 & 2 & 0 \\
\hline Feedback & 19 & 33 & 1 & 0 \\
\hline Recognition & 13 & 36 & 0 & 0 \\
\hline Autonomy & 7 & 42 & 3 & 1 \\
\hline Work balance & 16 & 32 & 4 & 1 \\
\hline Management contribution & 13 & 37 & 2 & 0 \\
\hline Sense of Responsibility & 24 & 36 & 0 & 0 \\
\hline Sense of belonging & 15 & 34 & 3 & 0 \\
\hline Equity & 10 & 45 & 1 & 0 \\
\hline Job security & 12 & 29 & 6 & 3 \\
\hline Self-organizing teams & 15 & 39 & 2 & 1 \\
\hline Eliminate Politics & 17 & 21 & 8 & 1 \\
\hline Right amount of documentation & 7 & 33 & 4 & 2 \\
\hline Tolerance to work & 62 & 4 & 0 & 0 \\
\hline Life Insurance & 29 & 37 & 0 & 0 \\
\hline Annual Award System & 56 & 10 & 0 & 1 \\
\hline Recreational tours & 50 & 15 & 1 & 0 \\
\hline Staff Dinner & 43 & 22 & 2 & 0 \\
\hline Leave on demand & 47 & 16 & 1 & 1 \\
\hline Recording suggestions & 40 & 26 & 1 & 0 \\
\hline Client Availability & 47 & 19 & 0 & 1 \\
\hline Recreational facility & 41 & 23 & 1 & 1 \\
\hline Follow standard Practices & 48 & 18 & 0 & 1 \\
\hline Managing Self respect & 57 & 9 & 1 & 0 \\
\hline Knowledgeable Team Leader & 57 & 8 & 2 & 0 \\
\hline
\end{tabular}




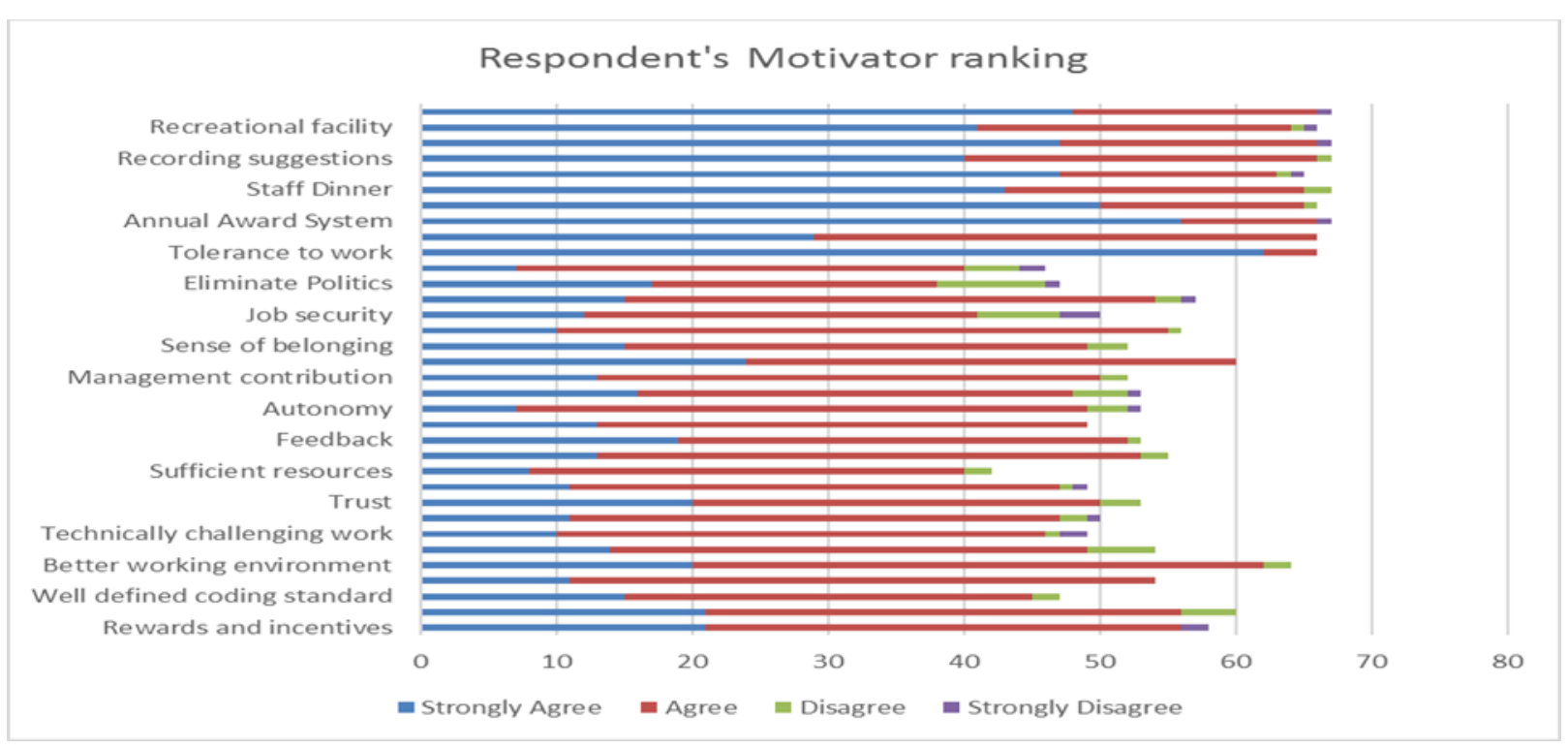

Fig. 7. Respondents motivators ranking.

Following Fig. 7 shows the respondents motivator ranking into more accurate graphical work in which tolerance to work has highest motivator value.

2) Demotivators respondents: Following are the respondent's ranking about the demotivator factors of agile software development (Table VII). The result indicates that lack of resources is the biggest demotivator factor among all factors while unrealistic goals are the least demotivator factor.

Following Fig. 8 shows the respondents demotivator ranking into more accurate graphical work in which lack of resources has highest demotivator value.

TABLE VII. RESPONDENTS DEMOTIVATOR RESULTS

\begin{tabular}{|c|c|c|c|c|}
\hline Demotivating Factors & Strongly Agree & Agree & Disagree & Strongly Disagree \\
\hline Communication Barrier & 24 & 29 & 7 & 0 \\
\hline Lack of relationship opportunities & 13 & 29 & 11 & 0 \\
\hline Unrealistic goals & 20 & 21 & 6 & 0 \\
\hline Injustice in Promotions & 15 & 29 & 8 & 2 \\
\hline Poor quality software & 13 & 23 & 10 & 4 \\
\hline Political Environment & 14 & 24 & 9 & 1 \\
\hline Uncompetitive pay & 16 & 24 & 12 & 0 \\
\hline Unsupportive management & 24 & 15 & 9 & 0 \\
\hline Lack of influence & 10 & 28 & 11 & 3 \\
\hline Unfair reward system & 19 & 27 & 8 & 1 \\
\hline Non-interesting work & 16 & 19 & 8 & 2 \\
\hline Inequity/Personal preferences & 12 & 29 & 7 & 0 \\
\hline Risk & 3 & 39 & 7 & 0 \\
\hline Stress/Pressure & 14 & 32 & 8 & 1 \\
\hline Less Documentation & 37 & 28 & 0 & 0 \\
\hline Restricted Social Networking & 36 & 31 & 0 & 1 \\
\hline Job threatening & 45 & 18 & 2 & 1 \\
\hline Lack of Resources & 47 & 20 & 0 & 0 \\
\hline Political Background & 36 & 30 & 1 & 0 \\
\hline Late Hours & 42 & 25 & 0 & 0 \\
\hline Sectarian Discrimination & 36 & 29 & 1 & 1 \\
\hline Lack of Team work & 37 & 28 & 0 & 0 \\
\hline Prohibition of change & 34 & 31 & 0 & 0 \\
\hline Long Term Project & 32 & 35 & 0 & 0 \\
\hline
\end{tabular}


Respondent's Demotivator factors

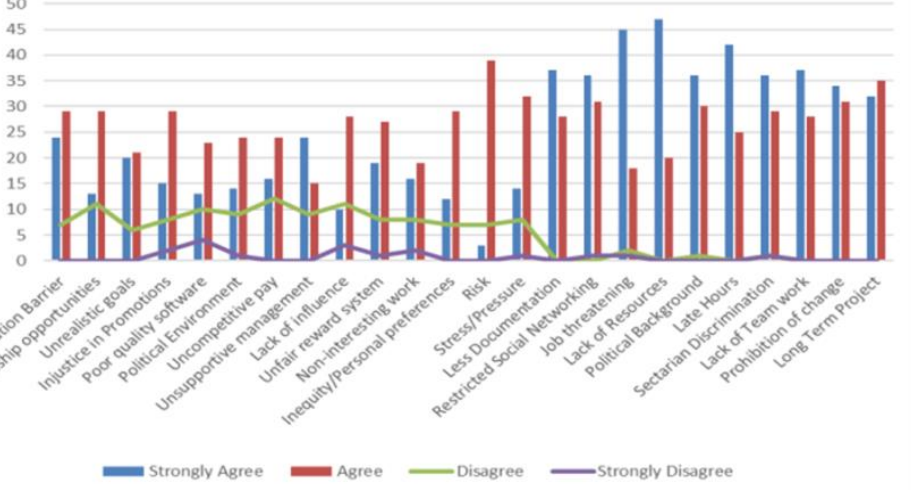

Fig. 8. Respondents demotivators ranking.

\section{G. Correlation Factors (Answering RQ\# 1)}

Following Table VIII shows the correlation between the motivator factors. The factor which has 0 to 0.25 value has weak positive correlation. The factors having value from 0.25 to 0.75 has medium positive correlation and the factor has more than 0.75 value has strongest positive correlation. Likewise, if the factor has 0 to -0.25 value has weakest negative correlation, if a factor has -0.25 to -0.75 value has medium negative correlation and if a function has less than 0.75 has strongest negative correlation.

TABLE VIII. CORRELATION BETWEEN MOTIVATOR FACTORS

\begin{tabular}{|c|c|c|c|c|c|c|c|c|c|c|c|c|c|c|c|c|c|c|c|c|c|c|c|c|c|c|c|c|c|c|c|c|c|c|c|c|}
\hline \multicolumn{37}{|c|}{ Correlations } \\
\hline & 3 & 恣 & 3 & 3 & 3 & a & 3 & 3 & 3 & 3 & 3 & 3 & $\stackrel{3}{\mathrm{~s}}$ & $\underset{\omega}{3}$ & $\frac{3}{b}$ & 光 & $\frac{3}{a}$ & 3 & $\underset{\infty}{3}$ & $\frac{3}{6}$ & 忐 & $\begin{array}{l}3 \\
\underline{N} \\
\end{array}$ & $\begin{array}{l}3 \\
\text { N } \\
\text { 促 }\end{array}$ & 胥 & $\begin{array}{l}3 \\
\mathbb{1} \\
+\end{array}$ & 孞 & 孞 & 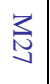 & $\underset{\infty}{3}$ & 芯 & 孞 & 录 & 3 & $\underset{\omega}{3}$ & 㥕 & $\underset{w}{\mathbf{w}}$ \\
\hline 3 & $\overrightarrow{\dot{8}}$ & $i_{+}$ & $\begin{array}{l}\text { in } \\
\text { un }\end{array}$ & i & ì & $\begin{array}{l}0 \\
\text { i } \\
\text { s }\end{array}$ & $\begin{array}{l}0 \\
\dot{\omega} \\
-1\end{array}$ & $i_{+}$ & 站 & i & 只 & $\begin{array}{l}\text { i } \\
\text { î }\end{array}$ & $\dot{\ddot{\omega}}$ & $\begin{array}{l}\stackrel{0}{1} \\
\dot{\omega}\end{array}$ & $\stackrel{\circ}{\omega}$ & $\stackrel{0}{6}$ & $i_{u}$ & $\stackrel{0}{0}$ & 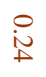 & i & $\begin{array}{l}0 \\
\doteq \\
\doteq\end{array}$ & $\begin{array}{l}0 \\
\vdots \\
=\end{array}$ & $\stackrel{\circ}{\stackrel{\circ}{N}}$ & $\begin{array}{l}\stackrel{1}{*} \\
\dot{f}\end{array}$ & $\begin{array}{l}0 \\
\dot{t}\end{array}$ & $\stackrel{0}{\Xi}$ & 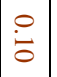 & $\begin{array}{l}\stackrel{0}{\sim} \\
\dot{\perp}\end{array}$ & $\begin{array}{l}0 \\
0 \\
0\end{array}$ & $\stackrel{0}{0}$ & $\begin{array}{l}0 \\
0 \\
0\end{array}$ & $\begin{array}{l}\dot{1} \\
\dot{w} \\
\text { ind }\end{array}$ & $\begin{array}{l}\dot{1} \\
\dot{g}\end{array}$ & $\stackrel{\circ}{\circ}$ & $\dot{8}$ & ஷ் \\
\hline 고 & & $\overrightarrow{8}$ & $\begin{array}{l}0 \\
\dot{w} \\
u\end{array}$ & i & $\mid \begin{array}{l}0 \\
\dot{n} \\
t\end{array}$ & $\stackrel{\circ}{\stackrel{\omega}{\omega}}$ & $\underset{\sim}{i}$ & 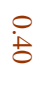 & i & : & $\begin{array}{l}0 \\
\dot{a} \\
\dot{a}\end{array}$ & $\begin{array}{l}0 \\
\dot{w} \\
u\end{array}$ & : & $\begin{array}{l}0 \\
\dot{4}\end{array}$ & $\stackrel{\circ}{\circ}$ & $\stackrel{\circ}{ \pm}$ & 离 & ن & i & $\begin{array}{l}0 \\
\dot{0} \\
\text { के }\end{array}$ & $\stackrel{0}{0}$ & $\stackrel{0}{\stackrel{\ddots}{\triangleleft}}$ & $\begin{array}{l}\circ \\
\dot{u}\end{array}$ & $\stackrel{0}{\stackrel{\infty}{\infty}}$ & $\stackrel{\ominus}{=}$ & $\begin{array}{l}\dot{1} \\
\dot{\infty}\end{array}$ & $\underset{\sim}{\dot{\omega}}$ & O̊ & $\begin{array}{l}\dot{1} \\
\dot{0} \\
\dot{0}\end{array}$ & $\stackrel{0}{\ddots}$ & $\begin{array}{l}\dot{1} \\
\dot{0} \\
\dot{u}\end{array}$ & $\begin{array}{l}\dot{b} \\
\dot{\omega}\end{array}$ & $\begin{array}{l}\dot{0} \\
\dot{D}\end{array}$ & $\begin{array}{l}\dot{1} \\
\dot{0} \\
\dot{a}\end{array}$ & $\stackrel{\circ}{\circ}$ & $\stackrel{8}{8}$ \\
\hline 3 & & & $\dot{\dot{\theta}}$ & $\dot{\omega}_{\sim}$ & $\mid \begin{array}{c}\dot{\omega} \\
\dot{+}\end{array}$ & 啰 & 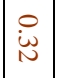 & 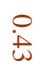 & in & 过 & 定 & $\begin{array}{l}\stackrel{i}{0} \\
\text { i }\end{array}$ & i. & $\begin{array}{l}\circ \\
\dot{\infty} \\
\infty\end{array}$ & 岕 & $\begin{array}{l}\circ \\
\dot{b}\end{array}$ & $\begin{array}{l}0 \\
\text { in } \\
u\end{array}$ & $\underset{\perp}{\stackrel{\sim}{\infty}}$ & i & 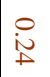 & 灾 & $\begin{array}{l}\dot{0} \\
\dot{\omega}\end{array}$ & $\begin{array}{l}\stackrel{i}{ \pm} \\
\stackrel{\perp}{*}\end{array}$ & $\underset{\dot{\omega}}{\stackrel{0}{0}}$ & $\frac{0}{N}$ & $\underset{i}{i}$ & $\stackrel{\circ}{\dot{u}}$ & $\stackrel{0}{0}$ & \begin{tabular}{|l|} 
\\
0 \\
0
\end{tabular} & $\stackrel{\circ}{\dot{\omega}}$ & i⿱ & $\stackrel{\circ}{\ominus}$ & $\begin{array}{l}0 \\
\dot{1}\end{array}$ & $\dot{\dot{q}}$ & $\begin{array}{l}0 \\
\dot{N} \\
\end{array}$ & $\dot{\circ}$ \\
\hline 3 & & & & $\dot{8}$ & $\underset{\infty}{i}$ & $\mid \begin{array}{l}0 \\
\text { i } \\
\text { d }\end{array}$ & $\mid \begin{array}{l}0 \\
\text { î }\end{array}$ & $\begin{array}{l}\stackrel{0}{t} \\
\stackrel{t}{0}\end{array}$ & i & 总 & $\mid \begin{array}{l}0 \\
\text { N } \\
\text { | }\end{array}$ & $\mid \begin{array}{l}0 \\
\dot{\omega} \\
\end{array}$ & $\underset{\omega}{\stackrel{\infty}{\omega}}$ & i & $\underset{\infty}{\stackrel{D}{\infty}}$ & $i_{\infty}$ & $\begin{array}{l}\stackrel{0}{\dot{U}} \\
\dot{v}\end{array}$ & ì & $\underset{\dot{\omega}}{\stackrel{\sim}{+}}$ & 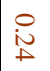 & $\left|\begin{array}{l}0 \\
\dot{u} \\
u\end{array}\right|$ & $\stackrel{0}{i}$ & $\begin{array}{l}\underset{\sim}{\infty} \\
\underset{\infty}{0}\end{array}$ & : & $\begin{array}{l}\dot{0} \\
\dot{0}\end{array}$ & $\stackrel{0}{\circ}$ & 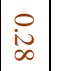 & $\begin{array}{l}\stackrel{0}{u} \\
\stackrel{u}{u}\end{array}$ & $\underset{\omega}{\dot{\omega}}$ & $\mid \begin{array}{l}0 \\
\dot{\perp}\end{array}$ & 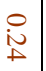 & $\begin{array}{l}0 \\
0 \\
0\end{array}$ & $\begin{array}{l}\dot{1} \\
\dot{q}\end{array}$ & $\stackrel{\circ}{\dot{1}}$ & $\begin{array}{l}\text { i } \\
\text { d }\end{array}$ & $\stackrel{\circ}{\stackrel{P}{P}}$ \\
\hline 3 & & & & & $\dot{\dot{8}}$ & 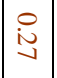 & $\begin{array}{l}\dot{0} \\
\dot{\sim} \\
\dot{O}\end{array}$ & in & in & $\stackrel{\circ}{\underset{\sim}{u}}$ & $\underset{\sim}{\stackrel{\sim}{\sim}}$ & $\begin{array}{l}0 \\
\text { i } \\
\infty\end{array}$ & $\begin{array}{l}\stackrel{1}{ \pm} \\
\pm\end{array}$ & $\begin{array}{l}\circ \\
\pm\end{array}$ & $\underset{\sim}{\dot{w}}$ & $\dot{\sim}_{\sim}$ & 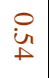 & $\begin{array}{l}\text { \& } \\
\text { tे }\end{array}$ & 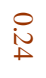 & 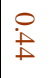 & $\begin{array}{l}\stackrel{0}{0} \\
\dot{\tilde{\sigma}}\end{array}$ & $\mid \begin{array}{l}0 \\
\text { 立 } \\
\text { 出 }\end{array}$ & 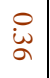 & $\underset{\sim}{\stackrel{\sim}{u}}$ & $\stackrel{\infty}{\infty}$ & $\begin{array}{l}\dot{2} \\
\dot{2}\end{array}$ & $\mid \begin{array}{l}\stackrel{i}{ \pm} \\
\stackrel{\sim}{*}\end{array}$ & $\begin{array}{l}\dot{0} \\
\dot{1}\end{array}$ & $\begin{array}{l}\dot{0} \\
\dot{0}\end{array}$ & $\stackrel{\circ}{\dot{\sim}}$ & $\stackrel{:}{:}$ & $\begin{array}{l}0 \\
0 \\
0\end{array}$ & : & $\dot{u}$ & $\ddot{8}$ & $\mathbb{y}$ \\
\hline a & & & & & & $\dot{\dot{8}}$ & $\mid \begin{array}{l}0 \\
\dot{1} \\
\infty\end{array}$ & $\stackrel{i}{u}_{u}$ & 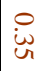 & $\begin{array}{l}0 \\
\dot{1} \\
\dot{\omega}\end{array}$ & $\stackrel{0}{=}$ & 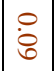 & $\begin{array}{l}\text { in } \\
\text { ind }\end{array}$ & $\stackrel{\circ}{\stackrel{\Xi}{*}}$ & 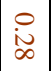 & 总 & $\begin{array}{l}0 \\
\dot{\infty} \\
\infty\end{array}$ & 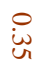 & $\stackrel{\ominus}{\perp}$ & $\begin{array}{l}\circ \\
\text { i } \\
\text { a }\end{array}$ & $\stackrel{0}{0}$ & $\begin{array}{l}0 \\
\text { î }\end{array}$ & $\begin{array}{l}0 \\
\dot{u} \\
u\end{array}$ & $\begin{array}{l}\stackrel{ }{\perp} \\
\perp\end{array}$ & $\stackrel{O}{\stackrel{0}{N}}$ & $\underset{⿱ ⺌}{0}$ & $\stackrel{0}{\circ}$ & $\begin{array}{l}\dot{1} \\
\dot{0}\end{array}$ & 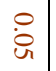 & $\begin{array}{l}\dot{d} \\
\dot{N}\end{array}$ & ؛े & $\begin{array}{l}\dot{1} \\
0 \\
0\end{array}$ & 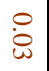 & $\begin{array}{l}i \\
i \\
\infty\end{array}$ & $\underset{\sim}{i}$ & $\underset{v}{\omega}$ \\
\hline 3 & & & & & & & 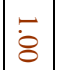 & $\underset{\perp}{\stackrel{i}{+}}$ & 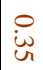 & $\begin{array}{l}0 \\
\stackrel{ \pm}{ \pm}\end{array}$ & $\mid \begin{array}{l}0 \\
\infty \\
\infty\end{array}$ & 离 & $\begin{array}{l}i \\
\dot{\omega}\end{array}$ & $\begin{array}{l}\stackrel{0}{0} \\
\dot{\alpha}\end{array}$ & $\begin{array}{l}\circ \\
\doteq\end{array}$ & : & $\begin{array}{l}\text { i } \\
\text { i }\end{array}$ & i & $\dot{\infty}$ & $\begin{array}{l}0 \\
\dot{h r} \\
t\end{array}$ & $\left|\begin{array}{l}\underset{1}{1} \\
\infty\end{array}\right|$ & $\mid \begin{array}{l}\stackrel{1}{1} \\
\dot{f}\end{array}$ & $\begin{array}{l}\underset{i}{+} \\
\perp\end{array}$ & 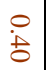 & $\stackrel{0}{0}$ & $\begin{array}{l}1 \\
0 \\
0\end{array}$ & 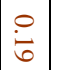 & $\begin{array}{l}\underset{i}{+} \\
\perp\end{array}$ & $\begin{array}{l}\circ \\
\dot{\sim} \\
\Omega\end{array}$ & $\begin{array}{l}\dot{1} \\
\dot{0}\end{array}$ & $\dot{d}$ & $\begin{array}{l}\dot{0} \\
\dot{\omega}\end{array}$ & $\stackrel{0}{\circ}$ & $\stackrel{8}{\circ}$ & $\stackrel{0}{\circ}$ & i \\
\hline$z_{\infty}$ & & & & & & & & $\dot{8}$ & in & in & $\begin{array}{l}\stackrel{0}{\perp} \\
\dot{+}\end{array}$ & $\begin{array}{l}0 \\
\dot{t} \\
\end{array}$ & $\underset{\sim}{\underset{\sim}{\sim}}$ & $\begin{array}{l}\circ \\
\dot{\perp}\end{array}$ & $\mid \begin{array}{l}0 \\
\dot{t}\end{array}$ & $\begin{array}{l}0 \\
\dot{t}\end{array}$ & $\mid \begin{array}{l}\dot{D} \\
\infty \\
\infty\end{array}$ & $\underset{\sim}{\dot{\omega}}$ & 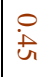 & $\stackrel{一}{\ddagger}$ & $\mid \begin{array}{l}\stackrel{0}{i} \\
+ \\
+\end{array}$ & 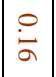 & 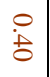 & 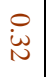 & $\stackrel{0}{\infty}$ & $\stackrel{0}{\circ}$ & $\stackrel{.}{i}$ & $\begin{array}{l}\dot{1} \\
\dot{\infty}\end{array}$ & $\begin{array}{l}0 \\
\dot{v} \\
\dot{v}\end{array}$ & $\because$ & $\stackrel{\circ}{*}$ & $\begin{array}{l}0 \\
\text { in } \\
\text { in }\end{array}$ & $\begin{array}{l}0 \\
\vdots \\
0\end{array}$ & $\stackrel{0}{a}$ & $\dot{0}$ & $\stackrel{0}{6}$ \\
\hline 3 & & & & & & & & & 8 & 竞 & 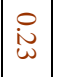 & $\stackrel{\dot{\omega}}{\bullet}$ & $\dot{\omega}_{0}$ & $\mid \begin{array}{c}\dot{\omega} \\
\dot{v}\end{array}$ & 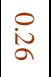 & i & 总 & $\dot{\omega}_{u}$ & 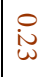 & $\stackrel{0}{\dot{u}}$ & $\stackrel{\stackrel{P}{\sim}}{\stackrel{\sim}{\sim}}$ & 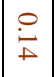 & $\begin{array}{l}\stackrel{D}{\sim} \\
\text { i }\end{array}$ & $\underset{i}{\stackrel{i}{*}}$ & $\underset{⿱ 亠 䒑}{\stackrel{.}{~}}$ & $\begin{array}{l}0 \\
\dot{0} \\
0\end{array}$ & $\stackrel{0}{\Xi}$ & $\stackrel{\circ}{\Xi}$ & 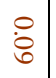 & $\begin{array}{l}0 \\
0 \\
0\end{array}$ & 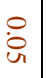 & $\begin{array}{l}\dot{0} \\
\dot{u} \\
u\end{array}$ & $\begin{array}{l}\dot{1} \\
\dot{u}\end{array}$ & $\begin{array}{l}\dot{1} \\
\dot{\omega}\end{array}$ & $\begin{array}{l}\dot{1} \\
\dot{0} \\
ن\end{array}$ & 0 \\
\hline$\frac{3}{0}$ & & & & & & & & & & $\dot{8}$ & $\mid \begin{array}{l}0 \\
\dot{f} \\
\end{array}$ & $\mid \begin{array}{l}\stackrel{.}{\omega} \\
+\end{array}$ & $\begin{array}{l}\stackrel{D}{ } \\
\dot{b}\end{array}$ & $\begin{array}{l}\dot{\tilde{O}} \\
\dot{O}\end{array}$ & $\mid \begin{array}{c}\stackrel{.}{\omega} \\
+\end{array}$ & 岕 & 品 & $\stackrel{\dot{\omega}}{.}$ & $\dot{\omega}_{\perp}$ & $\begin{array}{l}\stackrel{0}{\dot{b}} \\
\dot{\omega}\end{array}$ & 畜 & 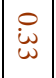 & $\stackrel{\ominus}{*}$ & $\underset{\infty}{\underset{\infty}{\infty}}$ & $\stackrel{\ominus}{=}$ & $\stackrel{\circ}{0}$ & $\mid \begin{array}{l}\dot{\omega}_{u} \\
\text { | }\end{array}$ & $\stackrel{\circ}{\circ}$ & $\stackrel{0}{\ominus}$ & 号 & $\stackrel{0}{:}$ & $\begin{array}{l}\dot{1} \\
\dot{u} \\
\dot{u}\end{array}$ & ì & $\stackrel{0}{\omega}$ & $\stackrel{0}{\vec{\not}}$ & $\dot{\omega}_{u}$ \\
\hline$\xi$ & & & & & & & & & & & $\dot{8}$ & 品 & 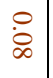 & $\left|\begin{array}{l}\underset{W}{\infty} \\
\infty\end{array}\right|$ & 该 & $\underset{\infty}{\infty}$ & î & 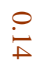 & ï & $\underset{\dot{\omega}}{\stackrel{\dot{\omega}}{ }}$ & $\dot{8}$ & $\begin{array}{l}1 \\
\dot{2} \\
\dot{1}\end{array}$ & $\begin{array}{l}\dot{1} \\
\dot{a}\end{array}$ & : : & : & $\begin{array}{l}\dot{b} \\
\dot{u} \\
0\end{array}$ & $\mid \begin{array}{l}\text { : } \\
\text { i্ }\end{array}$ & $\begin{array}{l}\dot{1} \\
\dot{\infty} \\
\infty\end{array}$ & $\stackrel{\circ}{\bar{\omega}}$ & ه̊ & : & $\begin{array}{l}0 \\
\text { in } \\
\text { in }\end{array}$ & $\stackrel{1}{\dot{1}}$ & $\begin{array}{l}\dot{i} \\
i \\
i=1\end{array}$ & 号 & $\ddot{\infty}$ \\
\hline & & & & & & & & & & & & $\overrightarrow{\dot{8}}$ & 㣽 & $\stackrel{\circ}{i}$ & $\begin{array}{l}0 \\
i \\
\infty \\
\infty\end{array}$ & i & i & $\stackrel{\omega}{\omega}$ & $\tilde{\alpha}$ & $\begin{array}{l}0 \\
\dot{f} \\
\text { U. }\end{array}$ & $\stackrel{0}{\stackrel{0}{N}}$ & $\begin{array}{l}\dot{1} \\
\dot{\omega} \\
\dot{u}\end{array}$ & @o & i & $\stackrel{\circ}{v}$ & $\stackrel{\dot{b}}{\not}$ & $\mid \begin{array}{l}0 \\
i \\
ن\end{array}$ & $\begin{array}{l}\dot{0} \\
\dot{N}\end{array}$ & $\stackrel{0}{\ominus}$ & $\stackrel{8}{8}$ & $\stackrel{\circ}{\ddot{u}}$ & $\mid \begin{array}{l}\dot{1} \\
\dot{w} \\
\sim\end{array}$ & & & & 8 \\
\hline
\end{tabular}




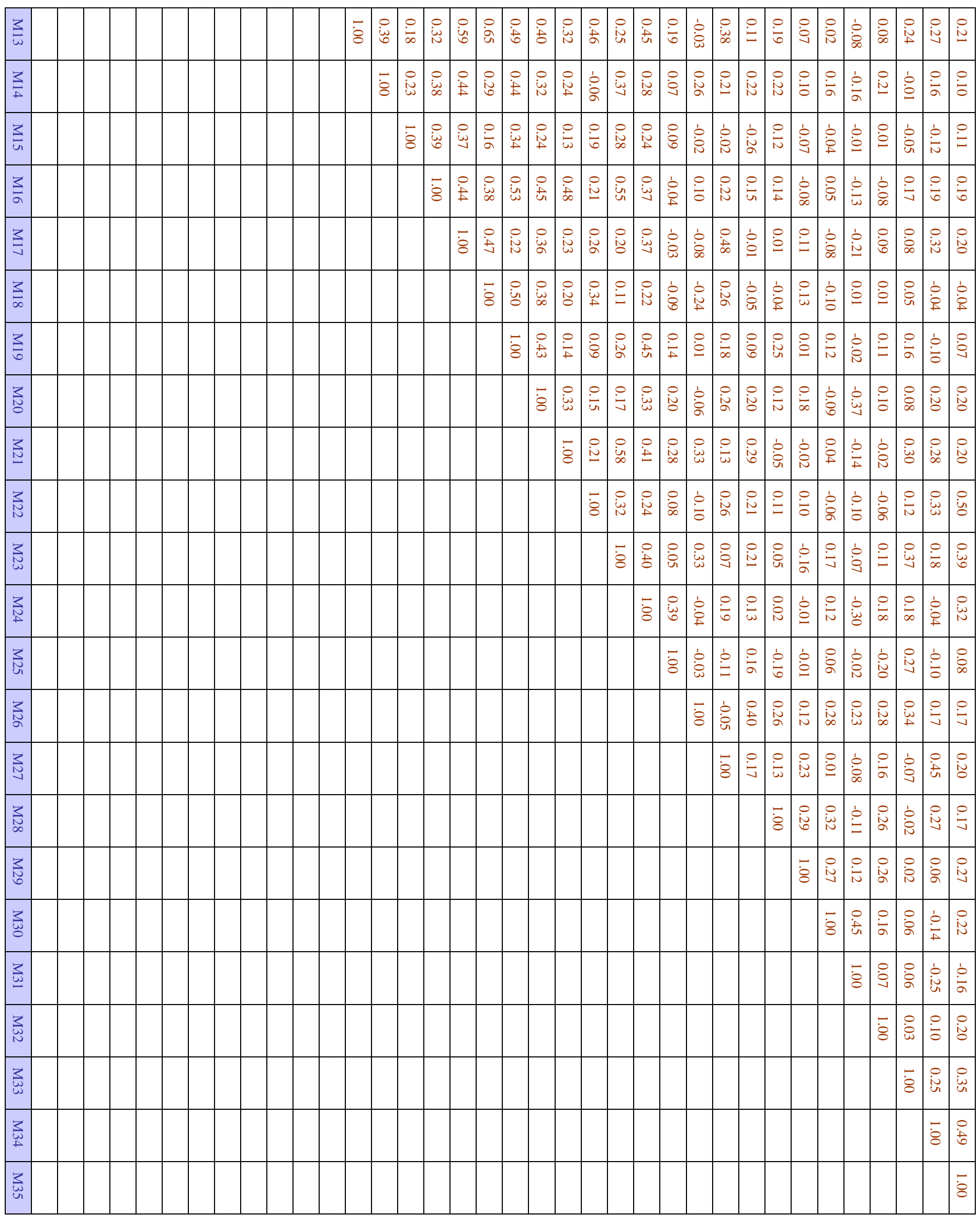


(IJACSA) International Journal of Advanced Computer Science and Applications,

Vol. 9, No. 7, 2018

\section{H. Comparison from Literature (Answering $R Q$ \# 2)}

This section provides the concrete information about the literature comparison with our survey. Based on the solid result a participant agreement and disagreement has been detailed discussed. Following Table IX shows the comparison between the findings in literature with our survey, respectively.

TABLE IX. MOTIVATORS FACTORS IN LITERATURE AND COMPARED WITH SURVEY

\begin{tabular}{|c|c|c|}
\hline Motivators from Literature & Motivators in this Survey & $\begin{array}{l}\text { Participants Agreement } \\
\text { / Disagreement with } \\
\text { Literature }\end{array}$ \\
\hline 1. "Working in company that is successful (e.g. financially stable)" [18] & $\begin{array}{l}\text { Working in successful company } \\
(47 / 67=70 \%)\end{array}$ & Strongly Agree \\
\hline $\begin{array}{l}\text { 2. "Good Management is cited } 3 \text { times as motivator due to the open } \\
\text { communication and workload balance in agile projects" [19] }\end{array}$ & $\begin{array}{ll}- & \text { Supportive management } \\
(56 / 67=83 \%)\end{array}$ & $\begin{array}{l}\text { Strongly } \\
\text { Agree }\end{array}$ \\
\hline $\begin{array}{l}\text { 3. "Factors unrelated to team interactions are not included, such as financial } \\
\text { compensation and job security. The findings we present here are all } \\
\text { based on how an individual's behaviour within a team might motivate or } \\
\text { de-motivate other developers." [20] }\end{array}$ & $\begin{array}{l}\text { Job security } \\
(41 / 67)=61 \%)\end{array}$ & Agree \\
\hline $\begin{array}{l}\text { 4. "we could conduct our experiment in a company under real working } \\
\text { conditions with employees of the company. Now, however, our internal } \\
\text { validity is threatened, because we cannot control the influence of } \\
\text { confounding variables like programming experience" [21] }\end{array}$ & $\begin{array}{l}\text { - Working with others/teamwork } \\
(62 / 67)=92 \%)\end{array}$ & Strongly Agree \\
\hline $\begin{array}{l}\text { 5. "Consistent with prior research individuals on both teams were } \\
\text { personally motivated by factors such as interesting and challenging } \\
\text { work, responsibility and the opportunity for growth and development as } \\
\text { part of a defined career path." [22] }\end{array}$ & $\begin{array}{l}\text { Career path } \\
(54 / 67)=80 \%)\end{array}$ & $\begin{array}{l}\text { Strongly } \\
\text { Agree }\end{array}$ \\
\hline $\begin{array}{l}\text { 6. "The allocation of office space, putting developers in close with each } \\
\text { other, the emphasis on face-toface communication, the availability of } \\
\text { appropriate development tools, and close customer collaboration require } \\
\text { a great deal of external support to be implemented" [23] }\end{array}$ & $\begin{array}{l}\text { Appropriate working conditions } \\
(60 / 67)=89 \%)\end{array}$ & Strongly Agree \\
\hline $\begin{array}{l}\text { 7. "Variety of work, the iteration planning meeting provides a forum in } \\
\text { which team members can easily and openly verbalize their preference to } \\
\text { work on specific task(s) in order to improve their knowledge and skills } \\
\text { in a certain area, which is motivating when, "people want areas of work } \\
\text { where they would learn the most... to acquire certain skills" [22] }\end{array}$ & $\begin{array}{l}\text { Variety of work } \\
(49 / 67)=73 \%)\end{array}$ & Agree \\
\hline $\begin{array}{l}\text { 8. "We ranked the motivators by their relative frequency in the results. The } \\
\text { most frequent general motivator we found is technically challenging } \\
\text { work (M1), in which work is not mundane and is technically } \\
\text { challenging" [19] }\end{array}$ & $\begin{array}{l}\text { - Technically challenging work } \\
(46 / 67=68 \%)\end{array}$ & Agree \\
\hline $\begin{array}{l}\text { 9. "Interestingly, based on responses to other questions, it does not seem to } \\
\text { matter whether the manager is perceived as actually understanding the } \\
\text { issues faced by practitioners, or whether rewards and incentives for } \\
\text { successful SPI are established" [24]. }\end{array}$ & $\begin{array}{l}\text { Rewards and incentives } \\
(56 / 67=83 \%)\end{array}$ & Strongly Agree \\
\hline $\begin{array}{l}\text { 10. "Trust/respect: All three agile practices were identified as an important } \\
\text { component of building trust in an agile team due to the increase in verbal } \\
\text { communication. In particular, the stand-up is a daily touch-point for all } \\
\text { team members, which requires team members (co-located and } \\
\text { distributed) to meet and communicate with each other on a daily basis } \\
\text { and "keeps the lines of communication open." }\end{array}$ & $\begin{array}{l}\text { Trust/respect } \\
(50 / 67=74 \%)\end{array}$ & Agree \\
\hline $\begin{array}{l}\text { 11. "The allocation of work in many agile teams and also in this team makes } \\
\text { it easy for developers to identify } \\
\text { 12. with tasks that have been fulfilled. The user story represents a task that } \\
\text { produces a visible part of the software." [25] }\end{array}$ & $\begin{array}{l}\text { - Identify with the task } \\
(47 / 67=70 \%)\end{array}$ & Agree \\
\hline $\begin{array}{l}\text { 13. "Limited supply of software engineers. Several sources2,3 have } \\
\text { indicated that the current US shortage of software personnel is between } \\
50,000 \text { and } 100,000 \text { people and that the suppliers (primarily university } \\
\text { computer science departments) do not have sufficient resources to meet } \\
\text { the future demand". [9] }\end{array}$ & $\begin{array}{l}-\quad \text { Sufficient resources } \\
\left(\begin{array}{ll}40 / 67=59 \quad \%\end{array}\right)\end{array}$ & Agree \\
\hline $\begin{array}{l}\text { 14. "For some, learning and development opportunities may have a higher } \\
\text { motivational impact, while for others compensation or supportive } \\
\text { superior may be more important". [26] }\end{array}$ & $\begin{array}{l}\text { - Development needs addressed } \\
(53 / 67=79 \%)\end{array}$ & $\begin{array}{ll}\text { b. } & \text { Strongly } \\
\text { c. } & \text { Agree }\end{array}$ \\
\hline $\begin{array}{l}\text { 15. "To make team-based performance evaluation more effective team } \\
\text { members can act as both evaluators and those being evaluated. Six } \\
\text { companies introduced } 360 \text {-degree feedback, in which all team members } \\
\text { evaluate one other (as opposed to managers appraising subordinates), } \\
\text { thus capturing voluntary contributions and mentorship".[27] }\end{array}$ & $\begin{array}{l}\text { - } \quad \begin{array}{l}\text { Feedback } \\
(52 / 67=77 \%)\end{array}\end{array}$ & $\begin{array}{ll}\text { d. } & \text { Strongly } \\
\text { e. } & \text { Agree }\end{array}$ \\
\hline $\begin{array}{l}\text { 16. "He concluded that recognition, security, and sense of belonging were } \\
\text { more important to productivity and morale or motivation, and a friendly } \\
\text { relationship with the supervisor was very important in securing the } \\
\text { loyalty and cooperation of the team" [28] }\end{array}$ & $\begin{array}{ll}-\quad & \text { Recognition } \\
& (49 / 67=73 \quad \%)\end{array}$ & Agree \\
\hline
\end{tabular}


17. "The motivating potential of a job is determined by the degree of richness of five core job dimensions: skill variety, task identity, task significance, autonomy and feedback from the job. The job's motivating potential score (MPS) is computed from the survey responses on the core job dimensions". [29]

18. "Project managers have to deal with peaking workloads, making it difficult to achieve a work-life balance. Particularly, the temporary nature of project work is a challenge for project managers. Often, there is an uncertainty about future assignments, including the nature of the assignment, its location, and future work colleagues" [26]

19. "In addition, factors such as career development, a sense of belonging and making a contribution to the entire system, receiving positive feedback, and having autonomy were also identified as important motivational factors for project managers" [26]

20. "Santana and Robey's (1995) model suggests that managerial, team member or self-control of tasks influences the level of job satisfaction felt by an employee. Two of these motivators are represented in the new model by 'good management', and 'empowerment/responsibility' but the notion of other team members controlling tasks is not explicitly mentioned". [18]

21. "factors such as career development , a sense of belonging and making a contribution to the entire system, receiving positive feedback" [26]

22. "Equity Theory (Homans and Adams in (Couger and Zawacki, 1980)) explains motivation in terms of matching the inputs that practitioners bring to a job (experiences, qualifications, etc.) with appropriate outputs (pay, responsibility, authority, etc.)." [16]

23. "trade-offs across sensor, networking, fusion, command-control, software infrastructure elements of a SISOS and more, along with additional trade-offs between performance, security, usability, safety, and fault tolerance" [30]

24. "Based on results of a survey with 1005 managers and technical employees in an insurance company" [26]

25. "Risk mitigation practices include career path development, mentoring junior staff to provide replacements for key personnel, incremental completion bonuses, flowdown of contract award fees to project performers, and recognition initiatives for valued contributions". [30]

26. <new Add Motivator 1>[31]

27. < new Add Motivator 2>[32]

28. <new Add Motivator 3>[33]

29. $\quad<$ new Add Motivator $4>[34]$

30. <new Add Motivator 5>[35]

31. <new Add Motivator 6>[36]

32. <new Add Motivator 7>[37]

33. <new Add Motivator 8>[38]

34. <new Add Motivator 9>[39]

35. <new Add Motivator 10>[40]

\section{RESEARCH CONTRIBUTION}

Literature review predicts that there is less work done on motivators and demotivators of agile software development and need a strong analysis that can increase the software performance and productivity. This research aims to provide solid background to agile practitioners to increase their satisfaction level by prioritizing their motivators factors. For this purpose, survey data analysis method is selected.

\begin{tabular}{|c|c|}
\hline $\begin{array}{ll}\text { - } & \text { Autonomy } \\
& (49 / 67=73 \%)\end{array}$ & g. $\quad$ Agree \\
\hline $\begin{array}{l}\text { - Work balance } \\
(48 / 67=71 \%)\end{array}$ & h. $\quad$ Agree \\
\hline 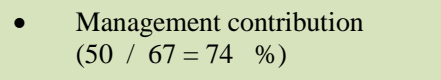 & i. $\quad$ Agree \\
\hline $\begin{array}{l}\text { - Sense of Responsibility } \\
(60 / 67=89 \%)\end{array}$ & $\begin{array}{ll}\text { j. } & \text { Strongly } \\
\text { k. } & \text { Agree }\end{array}$ \\
\hline $\begin{array}{ll}\text { - } & \text { Sense of belonging } \\
& (47 / 67=73 \%)\end{array}$ & Agree \\
\hline $\begin{array}{ll}- & \text { Equity } \\
(55 / 67=82 \%)\end{array}$ & $\begin{array}{l}\text { Strongly } \\
\text { Agree }\end{array}$ \\
\hline $\begin{array}{l}\text { Tolerance to work } \\
(66 / 67=98 \%)\end{array}$ & $\begin{array}{l}\text { Strongly } \\
\text { agree }\end{array}$ \\
\hline $\begin{array}{ll}- & \text { Life Insurance } \\
& (29 / 67=43 \%)\end{array}$ & Disagree \\
\hline $\begin{array}{l}\text { Annual Award System } \\
(66 / 67=98 \%)\end{array}$ & $\begin{array}{l}\text { Strongly } \\
\text { agree }\end{array}$ \\
\hline $\begin{array}{ll} & \text { Recreational tours } \\
(65 / 67=97 \%)\end{array}$ & $\begin{array}{l}\text { Strongly } \\
\text { agree }\end{array}$ \\
\hline $\begin{array}{l}\text { - } \quad \begin{array}{l}\text { Leave on demand } \\
(64 / 67=95 \%)\end{array}\end{array}$ & $\begin{array}{l}\text { Strongly } \\
\text { agree }\end{array}$ \\
\hline $\begin{array}{ll}\text { - } & \text { Recording suggestions } \\
(40 / 67=59 \%)\end{array}$ & Agree \\
\hline $\begin{array}{ll}- & \text { Client Availability } \\
& (66 / 67=98 \quad \%)\end{array}$ & Strongly disagree \\
\hline $\begin{array}{ll}- & \text { Follow standard Practices } \\
(66 / 67=98 & \%)\end{array}$ & Strongly Agree \\
\hline $\begin{array}{l}\text { - } \quad \begin{array}{l}\text { Knowledgeable Team Leader } \\
(54 / 67=80 \%)\end{array}\end{array}$ & $\begin{array}{l}\text { Strongly } \\
\text { Agree }\end{array}$ \\
\hline $\begin{array}{ll}\text { - } & \text { Managerial Politics } \\
& (17 / 67)=25 \%) \\
\end{array}$ & Strongly disagree \\
\hline $\begin{array}{l}\text { - } \quad \text { Right amount of documentation } \\
(33 / 67)=49 \%)\end{array}$ & Disagree \\
\hline $\begin{array}{ll} & \text { Staff dinner } \\
& (23 / 67)=34 \%)\end{array}$ & Strongly disagree \\
\hline $\begin{array}{ll}\text { - } & \text { Recreational facility } \\
& (41 / 67)=61 \%)\end{array}$ & Agree \\
\hline
\end{tabular}

\section{RESULTS AND DISCUSSION}

Prioritization of motivators and demotivators has been done by the help of software industrial survey. The main target of this research is to increase the motivation level of agile practitioners by increasing no of motivator and decreasing demotivator factors respectively. Our result indicates that, rewards and incentive and well-defined coding standard has strong correlation factors with value of 0.56 , while recreational 
tours has weakest correlation factors with value of -0.19 . In Management Supportive role, work load has highest correlation factors with 0.55 and staff dinner has weakest correlation factor. In well-defined coding standard, work load has highest correlation factor with 0.55 and job security is least correlation factor and vice versa. In comparison of our findings with literature, we have concluded that Knowledgeable team leader, leave on demands, tolerance to work, sense of responsibility and arranging recreational tours are the top motivators factors while staff dinner, life insurance and managerial politics are least motivating factors in agile software development.

Our result indicates that the participant responds tolerance to work as most strongly motivator factor, annual award system, manageable self-team and knowledgeable team leader are responds as other strong motivator factors. Besides the motivator factors, prioritization of demotivator factors has also been performed, lack of resources is the biggest demotivator factor while other strong demotivator factors include job threatening and late hours sittings. These findings lead to predict a guideline for agile practitioners that have strong impact on one's productivity.

\section{CONCLUSION AND FUTURE WORK}

This survey is conducted on 23 software companies of Pakistan who have implemented agile methods. There are total 67 agile practitioners who have participated in this research. The survey is the extended version of empirical research and case study of systematic mapping and literature review conducted on agile software developing. For this purpose, Pakistan a developing country is been chosen to evaluate our result. This research has revealed more motivator and demotivator factors than existing literature. The analysis has been done to find the top rank motivator and demotivator factors. Our result indicates that the tolerance to work is the highest motivator factor while managerial politics is the last. Likewise, lack of resources the most demotivator factor while the unrealistic goal is the least demotivator factor. These motivators and demotivator must be mitigated, in order to successful implementation of agile in their organizations.

The future work of this research us an implementation of model of motivator for agile practitioners. Another extension of this work is needed to find out motivator and demotivator factors according to core agile practices like planning iteration, iteration retrospective, daily stand ups and review meeting. By implementing motivator and demotivator factor on these agile practices we can attain more in-depth knowledge of this research.

\section{REFERENCES}

[1] A. Law and R. Charron, "Effects of agile practices on social factors," ACM SIGSOFT Softw. Eng. Notes, vol. 30, no. 4, p. 1, 2005.

[2] Z. Masood, R. Hoda, and K. Blincoe, "Motivation for Self-Assignment: Factors Agile Software Developers Consider," in 2017 IEEE/ACM 10th International Workshop on Cooperative and Human Aspects of Software Engineering (CHASE), 2017, pp. 92-93.

[3] O. Dieste, E. R. Fonseca C., G. Raura, and P. Rodriguez, "Professionals Are Not Superman: Failures beyond Motivation in Software Experiments," in 2017 IEEE/ACM 5th International Workshop on Conducting Empirical Studies in Industry (CESI), 2017, pp. 27-32.
[4] A. C. C. França, T. B. Gouveia, P. C. F. Santos, C. A. Santana, and F. Q. B. da Silva, "Motivation in software engineering: A systematic review update," 15th Annu. Conf. Eval. Assess. Softw. Eng. (EASE 2011), pp. 154-163, 2011.

[5] P. C. Chen, C. C. Chern, and C. Y. Chen, "Software project team characteristics and team performance: Team motivation as a moderator," in Proceedings - Asia-Pacific Software Engineering Conference, APSEC, 2012, vol. 1, pp. 565-570.

[6] A. Cockburn and J. Highsmith, "Agile software development: The people factor," Computer (Long. Beach. Calif)., vol. 34, no. 11, pp. 131-133, 2001.

[7] P. E. McMahon, "Bridging agile and traditional development methods: A project management perspective," CrossTalk, no. 5, pp. 16-20, 2004.

[8] M. Lindvall et al., "Empirical Findings in Agile Methods," Proc. Extrem. Program. Agil. Methods, XP/Agile Universe 2002, pp. 197207, 2002.

[9] B. Boehm and R. Turner, "Management challenges to implementing agile processes in traditional development organizations," IEEE Softw., vol. 22, no. 5, pp. 30-39, 2005.

[10] C. De O. Melo, C. Santana, and F. Kon, "Developers motivation in agile teams," Proc. - 38th EUROMICRO Conf. Softw. Eng. Adv. Appl. SEAA 2012, no. March 2015, pp. 376-383, 2012.

[11] J. Highsmith and A. Cockburn, "Agile Software Development: The Business of Innovation," Science (80-. )., vol. 34, no. 9, pp. 120-123, 2001.

[12] M. J. Akhtar, A. Ahsan, and W. Z. Sadiq, "Scrum adoption, acceptance and implementation (A case study of Barriers in Pakistan's IT Industry and Mandatory Improvements)," Proc. - 2010 IEEE 17th Int. Conf. Ind. Eng. Eng. Manag. IE EM2010, pp. 458-461, 2010.

[13] Colleen Frye, "Agile by the numbers: Survey finds more adoption, but age-old problems." [Online]. Available: http://searchsoftwarequality.techtarget.com/news/1372395/Agile-by-thenumbers-Survey-finds-more-adoption-but-age-old-problems. [Accessed: 24-Jul-2017].

[14] R. P. Wagener, "Investigating critical success factors in agile systems development projects/Ruhan Wagener.," no. November, 2012.

[15] T. Chow and D.-B. Cao, "A survey study of critical success factors in agile software projects," J. Syst. Softw., vol. 81, no. 6, pp. 961-971, 2008.

[16] N. Baddoo and T. Hall, "Motivators of Software Process Improvement: An analysis of practitioners' views," J. Syst. Softw., vol. 62, no. 2, pp. 85-96, 2002.

[17] I. Asghar and M. Usman, "Motivational and de-motivational factors for software engineers: An empirical investigation," Proc. - 11th Int. Conf. Front. Inf. Technol. FIT 2013, pp. 66-71, 2013.

[18] H. Sharp, N. Baddoo, S. Beecham, T. Hall, and H. Robinson, "Models of motivation in software engineering," Inf. Softw. Technol., vol. 51, no. 1, pp. 219-233, 2009.

[19] C. de O. Melo, C. Santana, and F. Kon, "Developers Motivation in Agile Teams," in 2012 38th Euromicro Conference on Software Engineering and Advanced Applications, 2012, pp. 376-383.

[20] S. Beecham, H. Sharp, N. Baddoo, T. Hall, and H. Robinson, "Does the XP environment meet the motivational needs of the software developer? An empirical study," in Proceedings - AGILE 2007, 2007, pp. 37-48.

[21] J. Feigenspan, C. Kästner, S. Apel, and T. Leich, "How to Compare Program Comprehension in FOSD Empirically - An Experience Report."

[22] O. Mchugh, K. Conoby, and M. Lang, "Motivating agile teams: A case study of teams in ireland and sweden," in 5th International Research Workshop on Information Technology Project Management (IRWITPM 2010), 2010, pp. 71-83.

[23] G. Asproni, "Motivation, Teamwork, and Agile Development," Agil. Times, vol. 4, no. 1, pp. 8-15, 2004.

[24] J. D. Herbsleb and D. R. Goldenson, "After the Apraissal: A systematic survey of CMM experience and results," pp. 323-330, 1996.

[25] B. Tessem and F. Maurer, "Job Satisfaction and Motivation in a Large Agile Team,” Lncs, vol. 4536, no. 5020, pp. 54-61, 2007. 
[26] S. Seiler, B. Lent, M. Pinkowska, and M. Pinazza, "An integrated model of factors influencing project managers' motivation - Findings from a Swiss Survey,” Int. J. Proj. Manag., vol. 30, no. 1, pp. 60-72, 2012.

[27] K. Conboy, S. Coyle, X. Wang, and M. Pikkarainen, "People over process: Key challenges in agile development," IEEE Softw., vol. 28, no. 4, pp. 48-57, 2011.

[28] L. Šteinberga and D. Šmite, "Towards Understanding of Software Engineer Motivation in Globally Distributed Projects," in 2011 IEEE Sixth International Conference on Global Software Engineering Workshop, 2011, pp. 117-119.

[29] J. D. Couger, V. Halttunen, and K. Lyytinen, "Evaluating the motivating environment in Finland compared to the United States - a survey," Eur. J. Inf. Syst., vol. 1, no. 2, pp. 107-112, 1991.

[30] A. Cockburn et al., "Advanced Software Technologies for Protecting America."

[31] D. Hutchison and J. C. Mitchell, Agile Processes in Software Engineering and Extreme Programming. 1973.

[32] S. Ahmed, K. Ghayyur, S. Ahmed, and A. Razzaq, "Motivators and Demotivators of Agile Software Development: Elicitation and Analysis," vol. 8, no. 12, pp. 304-314, 2017.

[33] M. Kropp and A. Meier, "Agile Success Factors A qualitative study about what makes agile projects successful," no. May 2015, 2015.
[34] S. Ahmed, K. Ghayyur, S. Ahmed, M. Ali, A. Razzaq, and N. Ahmed, "A Systematic Literature Review of Success Factors and Barriers of Agile Software Development," vol. 9, no. 3, pp. 278-291, 2018.

[35] A. C. C. Franca, D. E. S. Carneiro, and F. Q. B. da Silva, "Towards an Explanatory Theory of Motivation in Software Engineering: A Qualitative Case Study of a Small Software Company," 2012 26th Brazilian Symp. Softw. Eng., pp. 61-70, 2012.

[36] D. V Nithyanandan, "Work value as motivation among software professionals," Manag. Prudence J., vol. 1, no. 1, pp. 23-27, 2010.

[37] O. McHugh, K. Conboy, and M. Lang, "Using Agile Practices to Influence Motivation within IT Project Teams," Scand. J. Inf. Syst. (Special Issue IT Proj. Manag., vol. 23, p. pp 85-110, 2011.

[38] S. Misra, V. Kumar, U. Kumar, K. Fantazy, and M. Akhter, "Agile software development practices: evolution, principles, and criticisms," Int. J. Qual. Reliab. Manag., vol. 29, no. 9, pp. 972-980, 2012.

[39] A. Baird and F. J. Riggins, "Planning and Sprinting: Use of a Hybrid Project Management Methodology within a CIS Capstone Course," J. Inf. Syst. Educ., vol. 23, no. 3, pp. 243-257, 2012.

[40] D. Woit and K. Bell, "Do XP customer-developer interactions impact motivation? findings from an industrial case study," Proc. 7th Int. Work. Coop. Hum. Asp. Softw. Eng. - CHASE 2014, pp. 79-86, 2014.

\section{APPENDIX}

Fig. 9 shows the detailed list of motivators acronyms used in articles.

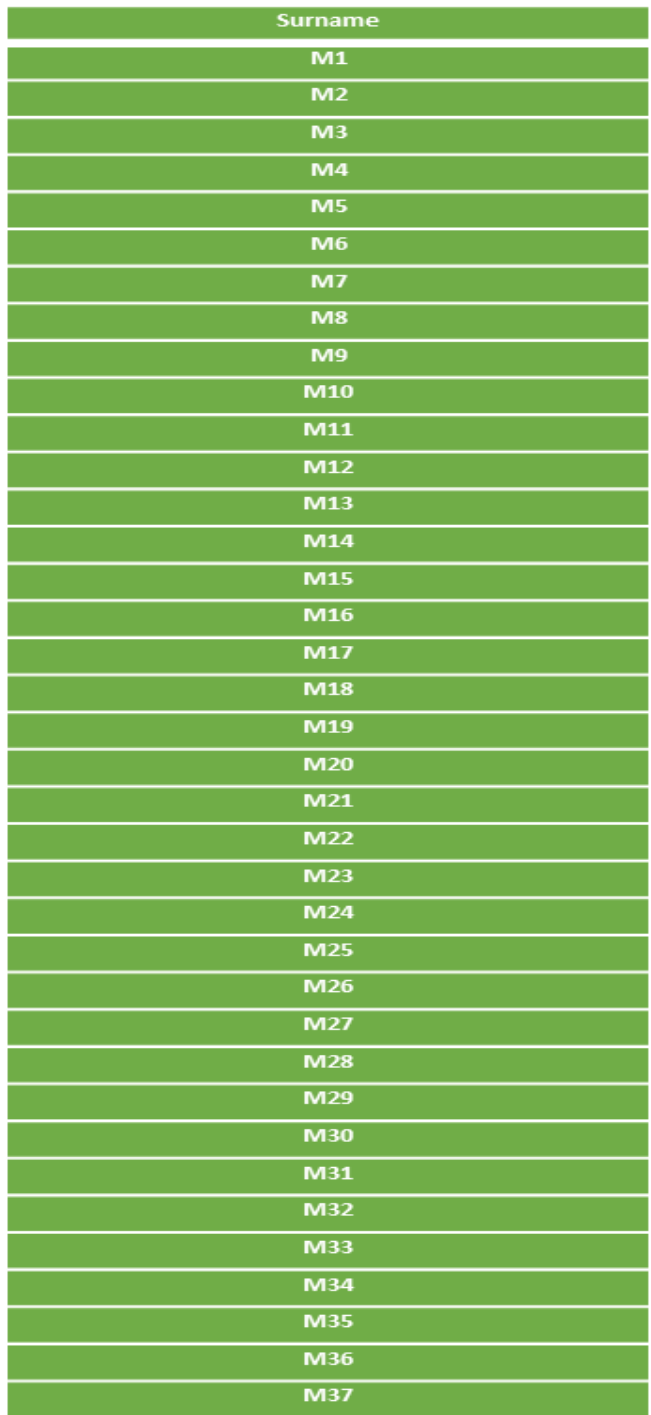

Fig. 9. Appendix. 